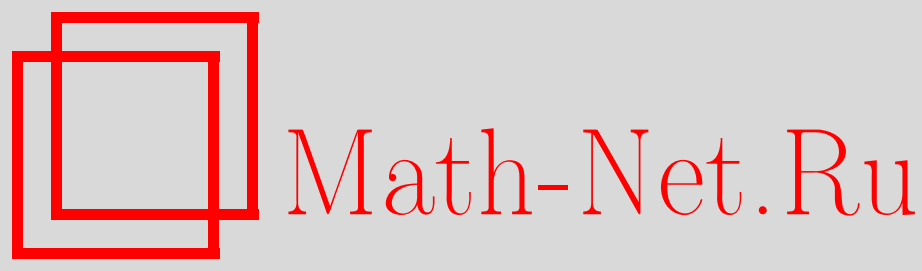

А. Визинеску, Д. Греку, Р. Феделе, С. Де Никола, Гидродинамический подход Маделунга к обобщенному нелинейному уравнению Шредингера с производной потенциала. Специальные решения и их устойчивость, $Т M \Phi$, 2009, том 160, номер 1, 229-239

DOI: https://doi.org/10.4213/tmf6394

Использование Общероссийского математического портала Math-Net.Ru подразумевает, что вы прочитали и согласны с пользовательским соглашением http: //www.mathnet.ru/rus/agreement

Параметры загрузки:

IP : 34.229 .45 .116

26 апреля 2023 г., 18:32:34

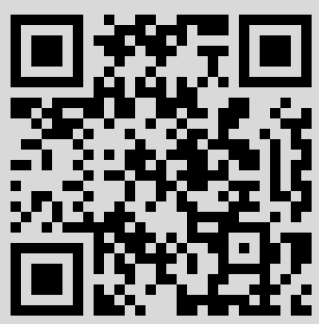




\title{
ГИДРОДИНАМИЧЕСКИЙ ПОДХОД МАДЕЛУНГА К ОБОБЩЕННОМУ НЕЛИНЕЙНОМУ УРАВНЕНИЮ ШРЕДИНГЕРА С ПРОИЗВОДНОЙ ПОТЕНЦИАЛА. СПЕЦИАЛЬНЫЕ РЕШЕНИЯ И ИХ УСТОЙЧИВОСТЬ
}

\begin{abstract}
$\mathrm{C}$ помощью гидродинамического описания Маделунга недавно удалось обнаружить соответствие между решениями обобщенного нелинейного уравнения Шредингера и решениями обобщенных уравнений КдФ. Аналогичным образом рассматривается специальное нелинейное уравнение Шредингера с производной потенциала. В случае движения с неизменным профилем вектора скорости потока найдено семейство решений в виде уединенных волн и периодические решения (выраженные через эллиптические функции Якоби). Устойчивость яркой солитонной моды (низшего состояния) исследуется в предположении о применимости критерия Вахитова-Колоколова.
\end{abstract}

Ключевые слова: нелинейное уравнение в частных производных, обобщенное нелинейное уравнение Шредингера, обобщенное уравнение КдФ, гидродинамический подход Маделунга.

\section{1. ВВЕДЕНИЕ}

В 1926 г. Маделунг предложил первое статистическое описание квантовой механики [1]. Если представить волновую функцию в виде $\Psi=\sqrt{\rho} e^{i \theta}$, то уравнение Шредингера в одномерном случае можно записать как

$$
i \hbar \frac{\partial \Psi}{\partial t}=-\frac{\hbar^{2}}{2 m} \frac{\partial^{2} \Psi}{\partial x^{2}}+m U(x) \Psi,
$$

при этом оно становится эквивалентным двум уравнениям на "плотность жидкости" $\rho=|\Psi|^{2}$ и “скорость жидкости" $v=(\hbar / m)(\partial \theta / \partial x)$ :

$$
\frac{\partial \rho}{\partial t}+\frac{\partial}{\partial x}(\rho v)=0
$$

*Department of Theoretical Physics, National Institute for Physics and Nuclear Engineering "Horia Hulubei", Bucharest, Romania. E-mail: avisin@theory.nipne.ro

${ }^{\dagger}$ University "Federico II, Naples", Naples, Italy

‡Institute of Cybernetics "Eduardo Caianello", Pozzuoli, Naples, Italy 


$$
\left(\frac{\partial}{\partial t}+v \frac{\partial}{\partial x}\right) v=\frac{\hbar^{2}}{2 m^{2}} \frac{\partial}{\partial x}\left(\frac{1}{\sqrt{\rho}} \frac{\partial^{2} \sqrt{\rho}}{\partial x^{2}}\right)-\frac{\partial U}{\partial x} .
$$

Первое из этих уравнений представляет собой уравнение непрерывности для плотности жидкости, а второе - уравнение движения для вектора потока жидкости. Интерпретация величины $v$ как скорости также следует из представления плотности потока через эти новые переменные:

$$
j=-i \frac{\hbar}{2 m}\left(\Psi^{*} \frac{\partial \Psi}{\partial x}-\Psi \frac{\partial \Psi^{*}}{\partial x}\right)=\rho v .
$$

В правой части уравнения (3), кроме обычного силового члена $\partial U / \partial x$, появляется квантовая составляющая $\frac{\hbar^{2}}{2 m^{2}} \frac{\partial}{\partial x}\left(\frac{1}{\sqrt{\rho}} \frac{\partial^{2} \sqrt{\rho}}{\partial x^{2}}\right)$, которая в литературе обычно называется потенциалом Бома.

В дальнейшем усилиями многих авторов подход Маделунга получил более наглядное объяснение (см. работы [2], [3] и приведенную в них литературу) и были предложены его многочисленные применения в различных областях, от стохастической квантовой механики [4] и квантовой космологии [5] до квантовых поправок к электронно-позитронной плазме [6], квантовомеханических эффектов в микроэлектронных приборах [7] и квантовых эффектов динамики пучков в ускорителях высоких энергий [8].

Некоторое время назад в работах [9] подход Маделунга был применен для вывода интересного соответствия между обобщенным нелинейным уравнением Шредингера (НУШ) и обобщенными уравнениями КдФ. Это соответствие справедливо по крайней мере для решений типа уединенной волны. В недавней работе [10] в рамках этого же подхода было исследовано обобщенное НУШ с производной потенциала. При интегрировании соответствующих обобщенных уравнений КдФ был получен обширный класс решений типа уединенных волн и периодических решений [11]. Также была обнаружена интересная связь между цилиндрическим НУШ и цилиндрическим уравнением КдФ [12].

Перечислим вкратце рассматриваемые нами уравнения.

Обобщенное НУШ [9]

$$
i \alpha \frac{\partial \Psi}{\partial t}+\frac{\alpha^{2}}{2} \frac{\partial^{2} \Psi}{\partial x^{2}}-U\left(|\Psi|^{2}\right) \Psi=0,
$$

где $U$ - некоторая функция от $|\Psi|^{2}$. При $U\left(|\Psi|^{2}\right) \propto|\Psi|^{2}$ уравнение (5) - это хорошо известное вполне интегрируемое НУШ. Интересен случай потенциала $U\left(|\Psi|^{2}\right)=$ $q_{1}|\Psi|^{2 p}+q_{2}|\Psi|^{4 p}$, отвечающего при $p=1$ нелинейности типа кубики-квинтики, которая имеет физически значимые применения.

Обобщенные НУШ с производной потенциала [10]

$$
\begin{aligned}
i \alpha \frac{\partial \Psi}{\partial t}+\frac{\alpha^{2}}{2} \frac{\partial^{2} \Psi}{\partial x^{2}}+i \beta \frac{\partial}{\partial x}\left(U\left(|\Psi|^{2}\right) \Psi\right) & =0 \\
i \alpha \frac{\partial \Psi}{\partial t}+\frac{\alpha^{2}}{2} \frac{\partial^{2} \Psi}{\partial x^{2}}+i \beta U\left(|\Psi|^{2}\right) \frac{\partial \Psi}{\partial x} & =0 .
\end{aligned}
$$


Уравнения (16) и (17) называются обобщенными уравнениями дНУШ-1 и дНУШ-2 соответственно. При $U\left(|\Psi|^{2}\right)=|\Psi|^{2}$ эти уравнения вполне интегрируемы [13], [14].

В настоящей работе рассматривается специальный класс обобщенных НУШ с производной:

$$
i \frac{\partial \Psi}{\partial t}+\frac{1}{2} \frac{\partial^{2} \Psi}{\partial x^{2}}+2 i \Psi^{2} \frac{\partial \Psi^{*}}{\partial x}+U\left(|\Psi|^{2}\right) \Psi=0
$$

где $U$ - некоторая функция от $|\Psi|^{2}$; при $U\left(|\Psi|^{2}\right)=4|\Psi|^{4}$ уравнение является вполне интегрируемым [15]. Особый характер уравнения (8) связан с формой нелинейного члена с производной $\Psi^{2} \partial \Psi^{*} / \partial x$, который отличен от похожего на него члена в уравнениях $(6),(7)$ и который не преобразуется к выражениям более общего вида. В следующем разделе будут представлены основные уравнения. В разделе 3 они интегрируются в случае стационарного профиля скорости потока. Легко находятся яркие солитоны и различные периодические решения. Последние становятся серыми (темными) солитонами в пределе $k \rightarrow 1$ ( $k$ - модуль эллиптической функции Якоби). В разделе 4 на основе хорошо известного критерия Вахитова-Колоколова (BK) [16] и более позднего подхода Кузнецова [17] к решению уравнений из широкого класса НУШ с производными обсуждается устойчивость ярких солитонов. Мы предполагаем, что критерий ВK применим к уравнению (8), которое, как оказалось, не было включено в широкий класс уравнений НУШ, рассмотренных в работах [16], [17], поэтому применимость результатов работ [16], [17] к уравнению (8) представляет на сегодняшний день открытую проблему. Наконец, некоторые замечания и выводы приведены в разделе 5 .

\section{2. ОСНОВНЫЕ УРАВНЕНИЯ}

Если подставить $\Psi=\sqrt{\rho} e^{i \theta}$ в уравнение (8), то после разделения вещественной и мнимой частей получаются уравнение непрерывности

$$
\frac{\partial \rho}{\partial t}+\frac{\partial}{\partial x}\left(\rho v+\rho^{2}\right)=0
$$

и уравнение движения

$$
\left(\frac{\partial}{\partial t}+v \frac{\partial}{\partial x}\right) v=\frac{1}{2} \frac{\partial}{\partial x}\left(\frac{1}{\sqrt{\rho}} \frac{\partial^{2} \sqrt{\rho}}{\partial x^{2}}\right)+2 \frac{\partial(v \rho)}{\partial x}+\frac{\partial U}{\partial x}
$$

где $\rho(x, t)=|\Psi|^{2}$ и $v(x, t)=\partial \theta(x, t) / \partial x$ суть соответственно плотность жидкости и ее скорость. Используя преобразования из работ [9], уравнение (10) можно привести к обобщенному уравнению КдФ

$$
\begin{aligned}
-\rho \frac{\partial v}{\partial t} & +v \frac{\partial \rho}{\partial t}+2\left[C_{0}(t)-\int \frac{\partial v}{\partial t} d x\right] \frac{\partial \rho}{\partial x}+ \\
& +\frac{1}{4} \frac{\partial^{3} \rho}{\partial x^{3}}+\left(\rho \frac{d U}{d \rho}+2 U\right) \frac{\partial \rho}{\partial x}+2 \rho^{2} \frac{\partial v}{\partial x}+4 v \frac{\partial \rho^{2}}{\partial x}=0
\end{aligned}
$$

где $C_{0}(t)-$ произвольная функция. 
Легко видеть, что не существует решения с постоянной скоростью потока. В самом деле, если $v=v_{0}$, то из уравнения непрерывности (9) получим

$$
\rho(x, t)=f\left[x-\left(v_{0}+2 \rho\right) t\right]
$$

где $f(x)=\rho(x, 0)$ - начальное условие. Данное решение несовместно с дисперсионным уравнением движения (10), и это является общим свойством НУШ с производной [10].

Таким образом, существуют только решения с непостоянным профилем скорости потока. Записав $\rho(x, t)=\rho(\xi), v(x, t)=v(\xi)$, где $\xi=x-u_{0} t$, и интегрируя (9), получим

$$
v=u_{0}-\rho+\frac{A_{0}}{\rho}
$$

где $A_{0}$ - постоянная интегрирования. Заметим, что функция $C_{0}(t)$ здесь становится произвольной константой $c_{0}$. Более того, $A_{0}=0$, если $\rho \rightarrow 0$ при $|\xi| \rightarrow \infty$ (яркие солитоны); для темных солитонов $\rho \rightarrow \rho_{0}, v \rightarrow v_{0}$ при $|\xi| \rightarrow \infty$, константа $A_{0}$ имеет вид $A_{0}=\rho_{0}\left(v_{0}-u_{0}+\rho_{0}\right)$. Уравнение типа КдФ (11) при этом записывается как

$$
\frac{1}{4} \frac{d^{3} \rho}{d \xi^{3}}+\left(\rho \frac{d U}{d \rho}+2 U\right) \frac{d \rho}{d \xi}+\left(2 c+u_{0}^{2}\right) \frac{d \rho}{d \xi}+3 u_{0} \frac{d \rho^{2}}{d \xi}-\frac{10}{3} \frac{d \rho^{3}}{d \xi}=0,
$$

где введена новая константа $c=c_{0}+3 A_{0}$. Уравнение (14) можно проинтегрировать при $U(\rho)=q \rho^{p}$ для $p=1,2$, а также в случае комбинации кубики-квинтики. В следующем разделе приводятся разнообразные решения уравнения (8) - уединенные волны, периодические решения, волны типа серых и темных солитонов.

\section{3. СПЕЦИАЛЬНЫЕ РЕШЕНИЯ}

3.1. Решения типа уединенных волн. Начнем список специальных решений уравнения (14) со случая $U(\rho)=q \rho^{2}$. Яркие солитоны получаются в качестве решений при $E=2 c+u_{0}^{2}=-b^{2}<0$ и $u_{0}>0$. При $q<5 / 2$, положив $\mu=(5-2 q) / 3$ и $\Delta=u_{0}^{2}-\mu b^{2}>0$, получим решение в виде

$$
\rho=\frac{b^{2}}{u_{0}+\sqrt{\Delta} \operatorname{ch}(2 b \xi)} .
$$

При $q>5 / 2$ решение имеет тот же вид (15), но с $\mu=(2 q-5) / 3$ и $\Delta=u_{0}^{2}+\mu b^{2}$. Напомним, что вполне интегрируемый случай $(q=4)$ отвечает $\mu=1$ и $\Delta=u_{0}^{2}+b^{2}$.

На основании формулы (15) легко вывести фазу $\theta(\xi)=\int\left(u_{0}-\rho\right) d \xi$. При $q<5 / 2$ имеем

$$
\theta(\xi)=u_{0} \xi+\frac{1}{\sqrt{\mu}} f(\xi)
$$

где

$$
f(\xi)=\frac{1}{2} \ln \frac{\sqrt{\Delta} e^{2 b \xi}+u_{0}+\sqrt{\mu} b}{\sqrt{\Delta} e^{2 b \xi}+u_{0}-\sqrt{\mu} b} .
$$


Легко видеть, что $f(\xi)$ монотонно убывает,

$$
f(-\infty)=\frac{1}{2} \ln \frac{u_{0}+\sqrt{\mu} b}{u_{0}-\sqrt{\mu} b}, \quad f(+\infty)=1 .
$$

При $q>5 / 2$

$$
f(\xi)=\operatorname{arctg} \frac{\sqrt{\Delta} e^{2 \xi b}+u_{0}}{\sqrt{\mu} b},
$$

эта функция также монотонно убывает, $f(-\infty)=\operatorname{arctg}\left(u_{0} / \sqrt{\mu} b\right), f(+\infty)=\pi / 2$.

Аналогично можно найти яркие солитоны при $U(\rho)=q \rho$ или при потенциале типа кубики-квинтики $U(\rho)=q_{1} \rho+q_{2} \rho^{2}$.

3.2. Периодические решения. Далее мы будем обсуждать периодические решения, причем будем рассматривать лишь интегрируемый случай $U=4 \rho^{2}$. Интегрируя дважды уравнение (14), получим

$$
\frac{1}{4}\left(\frac{d \rho}{d \xi}\right)^{2}=-P(\rho)
$$

где $P(\rho)=\rho^{4}+2 u_{0} \rho^{3}+E \rho^{2}+B \rho+D$, а $B$ и $D$ - константы.

Интересен случай, когда $P(\rho)$ имеет четыре различных корня, из которых хотя бы два положительны. Обозначим эти корни через $\rho_{4}<\rho_{3}<\rho_{2}<\rho_{1}$ и будем считать, что корни $\rho_{1}, \rho_{2}$ положительны. Напомним, что интерес представляет область значений $\rho_{2}<\rho<\rho_{1}$, в которой $-P(\rho)>0$. Тогда решение уравнения (17) записывается через эллиптический интеграл:

$$
\int_{\rho}^{\rho_{1}} \frac{d t}{\sqrt{\left(\rho_{1}-t\right)\left(t-\rho_{2}\right)\left(t-\rho_{3}\right)\left(t-\rho_{4}\right)}}=2 \xi .
$$

Результат выражается через эллиптическую функцию Якоби (см. справочник [18], формула 257.00):

$$
\rho=\rho_{4}+\frac{\rho_{1}-\rho_{4}}{1+m^{2} \mathrm{sn}^{2} u},
$$

где $\operatorname{sn}(u, k)$ - эллиптическая функция Якоби,

$$
\begin{aligned}
m^{2} & =\frac{\rho_{1}-\rho_{2}}{\rho_{2}-\rho_{4}}, & k^{2} & =\frac{\left(\rho_{1}-\rho_{2}\right)\left(\rho_{3}-\rho_{4}\right)}{\left(\rho_{1}-\rho_{3}\right)\left(\rho_{2}-\rho_{4}\right)}, \\
u & =\frac{2 \xi}{g}, & g & =\frac{2}{\sqrt{\left(\rho_{1}-\rho_{3}\right)\left(\rho_{2}-\rho_{4}\right)}} .
\end{aligned}
$$

Другой интересный случай имеет место, если все четыре вещественных корня оказываются положительными (тогда $\left.u_{0}<0\right)$ и $\rho \in\left(\rho_{4}, \rho_{3}\right)$. Решение выражается через интеграл:

$$
\int_{\rho_{4}}^{\rho} \frac{d t}{\sqrt{\left(\rho_{1}-t\right)\left(\rho_{2}-t\right)\left(\rho_{3}-t\right)\left(t-\rho_{4}\right)}}=2 \xi
$$

а результат записывается в виде

$$
\rho=\frac{\rho_{4}+\rho_{1} m^{2} \mathrm{sn}^{2} u}{1+m^{2} \operatorname{sn}^{2} u}
$$


где

$$
\begin{aligned}
u & =\frac{2 \xi}{g}, & g & =\frac{2}{\sqrt{\left(\rho_{1}-\rho_{3}\right)\left(\rho_{2}-\rho_{4}\right)}}, \\
m^{2} & =\frac{\rho_{3}-\rho_{4}}{\rho_{1}-\rho_{3}}, & k^{2} & =m^{2} \frac{\rho_{1}-\rho_{2}}{\rho_{2}-\rho_{4}} .
\end{aligned}
$$

Если найдена функция $\rho(\xi)$, то фазу $\theta(\xi)$ можно вычислить, используя соотношение

$$
\theta(\xi)=u_{0} \xi-\int \rho(\xi) d \xi+A_{0} \int \frac{d \xi}{\rho(\xi)}
$$

Здесь $A_{0}$ связана со значением скорости при $\xi=0$ : если $v(\xi)=v_{0}$ при $\xi=0$, то $A_{0}=\rho(0)\left[v_{0}-u_{0}+\rho(0)\right]$. Интегралы в выражении $(26)$ можно выразить через неполные эллиптические интегралы третьего рода

$$
\int_{0}^{u} \frac{d u^{\prime}}{1+m^{2} \operatorname{sn}^{2} u^{\prime}}=\Pi\left(u,-m^{2}\right)
$$

Например, в случае решения (24) получим

$$
\theta(\xi)=\left(u-\rho_{1}+\frac{A_{1}}{\rho_{1}}\right) \xi+\frac{g}{2}\left(\rho_{1}-\rho_{4}\right) \Pi\left(u,-m^{2}\right)+A_{1} \frac{\rho_{1}-\rho_{4}}{\rho_{1} \rho_{4}} \Pi\left(u,-m_{1}^{2}\right),
$$

где $m_{1}^{2}=\left(\rho_{1} / \rho_{4}\right) m^{2}$. Более сложный случай, который тем не менее может быть решен в терминах эллиптических функций Якоби, возникает, когда многочлен $P(\rho)$ имеет два несовпадающих положительных корня $\left(0<\rho_{2}<\rho<\rho_{1}\right)$ и два комплексно сопряженных корня $\left(\rho_{3}=\rho_{4}^{*}\right)$ (см. справочник [18], формула 259.00).

3.3. Предельные случаи. Рассмотрим теперь полученные решения в предельном случае, когда $k^{2} \rightarrow 1$ и sn $u \rightarrow$ th $u$. В обоих случаях, обсуждаемых выше, это происходит при $\rho_{2}=\rho_{3}$. Для решений из п. 3.1 соотношение $(21)$ принимает вид

$$
\rho=\rho_{4}+\frac{\rho_{1}-\rho_{4}}{1+m^{2} \operatorname{th}^{2}(2 \xi / g)}
$$

и описывает солитон типа яркого солитона, но с неисчезающими значениями на бесконечности $\left(\rho(\xi)=\rho_{1}\right.$ при $\xi=0$, в то время как $\rho(|\xi|) \rightarrow \rho_{2}$ при $\left.|\xi| \rightarrow \infty\right)$. Если $\rho_{2}=0$, то это решение переходит в солитон яркого типа, найденный ранее.

В случае решений из п. 3.2 соотношение (24) приобретает вид

$$
\rho=\frac{\rho_{4}+\rho_{1} m^{2} \operatorname{th}^{2} u}{1+m^{2} \operatorname{th}^{2} u}
$$

и описывает серый солитон $\left(\rho(0)=\rho_{4}, \rho(\infty)=\rho_{3}\right)$. При $\rho_{4}=0$ уравнение $(30)$ описывает решение типа темного солитона. 


\section{4. УСТОЙЧИВОСТЬ РЕШЕНИЙ}

Фундаментальным вопросом при исследовании решений нелинейных эволюционных уравнений является их устойчивость относительно малых возмущений. В дальнейшем мы ограничим рассмотрение возмущениями, которые сохраняют симметрию уравнения, т.е. имеют ту же размерность, что и само решение. Для обобщенного НУШ

$$
i \frac{\partial \Psi}{\partial t}+\frac{\partial^{2} \Psi}{\partial x^{2}}+f(|\Psi|) \Psi=0
$$

устойчивость его (ярких) солитонных решений исследовалась в рамках различных подходов (см. работы [16], [19]-[21] и статью [22], в которой приведен обзор последних результатов в трехмерном случае). Наиболее устойчивым должно быть основное состояние, которое для уравнения (31) имеет вид

$$
\Psi=e^{i \omega t} \Phi(x, \omega)
$$

с вещественнозначной функцией $\Phi(x, \omega)$. Критерий устойчивости был сформулирован Вахитовым и Колоколовым в работе [16]. Положив

$$
N(\omega)=\int \Phi^{2}(x, \omega) d x
$$

получим, что (яркое) основное состояние теряет устойчивость, если выполняется "условие наклона"

$$
\frac{d N(\omega)}{d \omega}<0
$$

Доказательство критерия BK [16], [19], [20] использует линеаризацию задачи в окрестности основного состояния и сводится к исследованию задачи на собственные значения для оператора, представляющего собой произведение двух линейных операторов Шредингера, один из которых в случае уравнений типа НУШ положительно определен, а другой имеет одно отрицательное собственное значение. Это свойство играет ключевую роль при доказательстве критерия ВК. Интересный и физически значимый анализ, выходящий за рамки линейного приближения, был произведен в работе [22], в которой в случае почти адиабатической и медленно меняющейся во времени неустойчивости было получено нелинейное дифференциальное уравнение второго порядка для $\omega(t)$ и было найдено несколько сценариев эволюции неустойчивости. Впоследствии подход к исследованию широкого класса НУШ с производными был развит Кузнецовым [17].

Критерий ВК применялся для классов уравнений типа НУШ, среди которых нет уравнения (8), и вопрос использования этого критерия в случае уравнения (8) на сегодняшний день остается открытым. Отметим, что для уравнения (8) соответствующая линеаризованная задача не сводится к задаче на собственные значения, как это происходит в случае мультипликативного оператора (см. детали в работе [17]).

С другой стороны, подход Кузнецова применим к широкому классу НУШ с производными, который также, к сожалению, не включает в себя уравнение (8), и вопрос о применимости к нему подхода Кузнецова тоже остается открытым. 
Таким образом, очевидно, что прямое применение обоих методов требует дополнительных доказательств. Тем не менее в случае критерия ВК можно с определенной степенью уверенности "предположить", что если ограничить рассмотрение солитоноподобными стационарными состояниями (в частности, основным состоянием), то этот критерий будет справедлив и для уравнения (8), так как он имеет простое физическое объяснение. В самом деле, видно, что, представив скорость в виде $V(x, t)=v(x, t)+\rho(x, t)=\partial \theta(x, t) / \partial x+\rho(x, t)$, мы можем привести уравнения (9), (10) к виду

$$
\begin{aligned}
\frac{\partial \rho}{\partial t}+\frac{\partial}{\partial x}(\rho V) & =0 \\
\left(\frac{\partial}{\partial t}+V \frac{\partial}{\partial x}\right) V & =\frac{1}{2} \frac{\partial}{\partial x}\left(\frac{1}{\sqrt{\rho}} \frac{\partial^{2} \sqrt{\rho}}{\partial x^{2}}\right)-\frac{\partial U^{\prime}}{\partial x},
\end{aligned}
$$

где $U^{\prime}=U^{\prime}(x, t)=U[\rho(x, t)]+2 V(x, t) \rho-(5 / 2) \rho^{2}(x, t)$. Стационарные решения уравнения (8) отвечают условию $V=V_{0}=$ const, которое эквивалентно условию $\partial \theta(x, t) / \partial x+\rho(x, t)=V_{0}$. Заметим, что в рамках гидродинамического представления Маделунга $V$ представляет собой "подходящую" скорость потока (см. уравнение (34)). Соответственно, ограничивая наш анализ только стационарными состояниями, получим, что два уравнения (34), (35) эквивалентны стационарному НУШ с мультипликативным нелинейным оператором потенциальной энергии

$$
U^{\prime}(x, t)=U\left[|\Psi(x, t)|^{2}\right]+2 V_{0}|\Psi(x, t)|^{2}-\frac{5}{2}|\Psi(x, t)|^{4} .
$$

Отсюда можно заключить, что стационарные решения НУШ такого типа отвечают стационарным решениям уравнения (8). В частности, основное состояние получается из условия $V_{0}=0$ (т.е. $\left.\partial \theta(x, t) / \partial x+\rho(x, t)=0\right)$. Аналогичное соответствие имеет место и в случае задачи об устойчивости стационарных состояний.

Апостериори мы можем дать ответ на вопрос, справедливо это предположение или нет. Для этого возьмем здесь и далее $U\left(|\Psi|^{2}\right)=q|\Psi|^{2}$ и применим критерий ВК для исследования основного состояния уравнения (8). Рассмотрим стационарное (основное) состояние вида

$$
\Psi(x, t)=e^{i \omega t} e^{i \Theta(x, \omega)} \Phi(x, \omega),
$$

в котором обе функции $\Theta(x, \omega)$ и $\Phi(x, \omega)$ вещественнозначны. Подставляя $(36)$ в $(8)$ и разделяя вещественную и мнимую части, получим

$$
\frac{d \Theta}{d x}=-\Phi^{2}
$$

и (после однократного интегрирования) имеем

$$
\left(\frac{d \Phi}{d x}\right)^{2}=\omega \Phi^{2}-q \Phi^{4}+\frac{5}{3} \Phi^{6} .
$$

Решение последнего уравнения имеет вид $(q>0, \omega>0)$

$$
\Phi=\frac{\sqrt{\omega}}{\sqrt{q+\sqrt{\Delta} \operatorname{ch}(2 \sqrt{\omega} x)}},
$$


где величина $\Delta=q^{2}-5 \omega / 3$ предполагается положительной, $q \sqrt{3 / 5 \omega}>1$. Фаза $\Theta(x, \omega)$ легко вычисляется из $(37)$ :

$$
\Theta(x, \omega)=\sqrt{\frac{3}{5}} \operatorname{arccth}\left(\sqrt{\frac{3 \Delta}{5 \omega}}\right)\left(e^{2 \sqrt{\omega} x}+\frac{q}{\sqrt{\Delta}}\right) .
$$

Данная функция монотонно убывает по $x$,

$$
\Theta(-\infty, \omega)=\sqrt{\frac{3}{5}} \operatorname{arccth}\left(q \sqrt{\frac{3}{5 \omega}}\right), \quad \Theta(+\infty, \omega)=0 .
$$

Это с очевидностью следует из (37), поскольку производная $\partial \Theta / \partial x$ всюду отрицательна. К правой части выражения (40) следует добавить положительную постоянную $\Theta_{0}$, чтобы обеспечить положительность $\Theta(x, \omega)$ на всей вещественной оси, и при этом решение $\Psi(x, t)$, задаваемое выражением (36), вполне может соответствовать вещественнозначному основному состоянию.

С учетом (39) можно легко вычислить величину $N(\omega)$ :

$$
N(\omega)=-(\Theta(+\infty, \omega)-\Theta(-\infty, \omega))=\sqrt{\frac{3}{5}} \operatorname{arccth}(z(\omega)), \quad z(\omega)=\sqrt{\frac{3}{5}} q \frac{1}{\sqrt{\omega}} .
$$

Поскольку $\frac{d z(\omega)}{d \omega}<0$ и $\frac{d(\operatorname{arccth} z)}{d z}<0$, очевидно, что $\frac{d N(\omega)}{d \omega}=\frac{d N(z)}{d z} \frac{d z}{d \omega}>0$, и в соответствии с критерием ВK стационарное состояние (39) устойчиво.

\section{5. ЗАКЛЮЧЕНИЯ И ЗАМЕЧАНИЯ}

Можно сделать два основных вывода.

Во-первых, отметим полезность подхода Маделунга при установлении соответствия между обобщенным НУШ с производной (8) и обобщенным уравнением КдФ. Если профиль скорости потока $v$ постоянен, то последнее уравнение легко интегрируется, что позволяет построить яркие, серые и темные солитонные решения, равно как и периодические решения. Насколько известно авторам, некоторые из этих решений не встречались ранее в литературе, что подчеркивает ценность предлагаемого подхода. Интересно, что гидродинамическое представление Маделунга было также использовано в настоящей работе для объяснения гипотезы о возможности применения критерия ВK при исследовании стационарных решений уравнения (8).

Во-вторых, мы рассмотрели проблему устойчивости основного состояния относительно малых возмущений. Применяя критерий BK, можно доказать, что основное состояние устойчиво, что оправдывает апостериори наше предположение о применимости критерия ВK к уравнению (8).

Авторам неизвестно ни одного строгого доказательства применимости критерия BK в случае обобщенного НУШ с производной. Следует отметить, что различие между обычным обобщенным НУШ и обобщенным НУШ с производной становится понятным с самого начала из определений стационарных состояний (32), (36). В случае обобщенного НУШ с производной необходимо вводить фазовую составляющую $\Theta(x, \omega)$, что затрудняет дальнейшие математические рассуждения. Вместо 
того чтобы исследовать свойства вещественнозначной функции $\Phi(x, \omega)$, необходимо вводить систему с двумя компонентами - вещественной и мнимой частями функции $\Psi$, при этом все уравнения становятся $(2 \times 2)$-матричными уравнениями. Но все же и в этом случае, как и в случае обобщенного НУШ, линеаризация нелинейного уравнения содержит голдстоуновскую моду, отвечающую нулевому собственному значению. Наличие этой моды представляет собой ключевой пункт в доказательстве критерия BK, и поэтому нам представляется достаточно вероятным, что должно существовать строгое доказательство справедливости выдвинутой нами гипотезы также и в случае обобщенного НУШ с производной.

С другой стороны отметим, что в настоящее время неизвестен способ прямого применения подхода Кузнецова к анализу устойчивости в случае НУШ с производной (8). Возможность применения этого подхода, а также строгое доказательство этой применимости в данном случае остается открытой задачей. Тем не менее на основе физических рассуждений, сходных с теми, которые были использованы в процессе применения критерия ВК, можно заключить, что подход Кузнецова применим к исследованию стационарных солитоноподобных решений уравнения (8). В дальнейших исследованиях мы надеемся расширить рамки этих методов и рассмотреть более сложные сценарии поведения систем, например систем с нелинейностью типа кубики-квинтики.

Благодарности. Авторы благодарны рецензенту за тщательное прочтение работы и сделанные при этом ценные замечания и предложения. Работа была проведена в рамках 15-й итальяно-румынской программы по науке и технологиям. Работа А. Визинеску и Д. Греку частично поддержана CNCSIS Program IDEI-325/2007, Pyмыния.

\section{Список литературы}

[1] E. Madelung, Z. Phys., 40 (1926), 332-326.

[2] D. Bohm, Phys. Rev., 85:2 (1952), 166-179.

[3] D. Bohm, B. J. Hiley, P. N. Kaloyerou, Phys. Rep., 144:6 (1987), 321-375.

[4] G. Auletta, Foundation and Interpretation of Quantum Mechanics, World Sci., Singapore, 2000.

[5] J. C. Vink, Nucl. Phys. B, 369:3 (1992), 707-728; N. Pinto-Neto, Found. Phys., 35:4 (2005), $577-603$.

[6] F. Haas, L. G. Garcia, J. Goedert, G. Manfredi, Phys. Plasmas, 10:10 (2003), 3858-3866; F. Haas, G. Manfredi, M. Feix, Phys. Rev. E, 62:2 (2000), 2763-2772.

[7] P. A. Markowich, C. A. Ringhofer, C. Schmeiser, Semiconductor Equations, Springer, Berlin, 1990.

[8] R. Fedele, D. Anderson, M. Lisak, Eur. Phys. J. B, 49:3 (2006), 275-281.

[9] R. Fedele, Phys. Scripta, 65:6 (2002), 502-508; R. Fedele, H. Schamel, Eur. Phys. J. B, 27:3 (2002), 313-320; R. Fedele, H. Schamel, P. K. Shukla, Phys. Scripta, T98:1 (2002), 18-23; R. Fedele, H. Schamel, V. I. Karpman, P. K. Shukla, J. Phys. A, 36:4 (2003), 1169-1173.

[10] D. Grecu, A. T. Grecu, A. Visinescu, R. Fedele, S. De Nicola, J. Nonlinear Math. Phys., 15, Suppl. 3 (2008), 209-219.

[11] A. Visinescu, R. Fedele, D. Grecu, S. De Nicola, "Periodic solutions of NLS equation in Madelung fluid description", Talk of International Conference on Fundamental and Applied Research in Physics FARPhys 2007 (25-28 October, Iassy, Romania), 2007. 
[12] R. Fedele, S. De Nicola, D. Grecu, P. K. Shukla, A. Visinescu, "Cylindrical nonlinear Schrödinger equation versus cylindrical Korteweg-de Vries equation", Frontiers in Modern Plasma Physics, AIP Conf. Proc., 1061, eds. P. K. Shukla, B. Eliasson, L. Stenflo, Melville, NY, 2008, 273-281.

[13] D. J. Kaup, A. C. Newell, J. Math. Phys., 19:4 (1978), 798-801; T. Kawata, H. Inoue, J. Phys. Soc. Japan, 44:6 (1978), 1968-1976; X.-J. Chen, J. Yang, W. K. Lam, J. Phys. A, 39:13 (2006), 3263-3274.

[14] A. Nakamura, H. H. Chen, J. Phys. Soc. Japan, 49:2 (1980), 813-816.

[15] M. J. Ablowitz, A. Ramani, H. Segur, J. Math. Phys., 21:5 (1980), 1006-1015; V. S. Gerdjikov, M. I. Ivanov, Bulgar. J. Phys., 10:2 (1983), 130-143; T. Tsuchida, J. Phys. A, 35:36 (2002), 7827-7847.

[16] Н. Г. Вахитов, А. А. Колоколов, Изв. вузов. Сер. радиофизика, 16:7 (1973), 1020-1028.

[17] Е. А. Кузнецов, ЖЭЭТФ, 116:1 (1999), 299-317.

[18] P. F. Byrd, M.D. Friedman, Handbook of Elliptic Integrals for Engineers and Scientists, Grundlehren Math. Wiss., 67, Springer, Berlin, 1971.

[19] E. A. Kuznetzov, A. M. Rubenchik, V. E. Zakharov, Phys. Rep., 142:3 (1986), 103-165.

[20] M. I. Weinstein, Comm. Pure Appl. Math., 39:1 (1986), 51-67.

[21] S. Cuccagna, "A survey on asymptotic stability of ground states of nonlinear Schrödinger equations", Dispersive Nonlinear Problems in Mathematical Physics, Quad. Mat., 15, ed. P. D’Ancona V. Georgev, Seconda Univ. Napoli, Caserta, 2004, 21-57.

[22] D. E. Pelinovsky, V. V. Afanasjev, Yu.S. Kivshar, Phys. Rev. E, 53:2 (1996), 1940-1953. 